JHR

36,1

Received 9 March 2020

Revised 30 August 2020 Accepted 29 September 2020

\section{Effects of an integrated intervention program for alcoholism (IIPA) on learning, memory and quality of life (QOL) in persons with alcohol dependence at the Centre for Addiction Medicine, Bengaluru, India}

\author{
Rajesh Kumar and Keshav J. Kumar \\ Clinical Psychology, National Institute of Mental Health and Neuro Sciences, \\ Bangalore, India \\ Vivek Benegal
}

Psychiatry, National Institute of Mental Health and Neuro Sciences, Bangalore, India Bangalore N. Roopesh

Clinical Psychology, National Institute of Mental Health and Neuro Sciences,

Bangalore, India, and

Girikematha S. Ravi

Biostatistics, National Institute of Mental Health and Neuro Sciences,

Bangalore, India

\begin{abstract}
Purpose - This study aims to examine the effectiveness of an integrated intervention program for alcoholism (IIPA) for improving verbal encoding and memory, visuospatial construction, visual memory and quality of life $(\mathrm{QoL})$ in persons with alcohol dependence.
\end{abstract}

(C) Rajesh Kumar, Keshav J. Kumar, Vivek Benegal, Bangalore N. Roopesh and Girikematha S. Ravi. Published in Journal of Health Research. Published by Emerald Publishing Limited. This article is published under the Creative Commons Attribution (CC BY 4.0) licence. Anyone may reproduce, distribute, translate and create derivative works of this article (for both commercial and non-commercial purposes), subject to full attribution to the original publication and authors. The full terms of this licence may be seen at http://creativecommons.org/licences/by/4.0/legalcode

The authors gratefully acknowledge the financial support (junior/senior research fellowship; Ref: $3 /$ 1/3JRF-2011/HRD-104) from the Indian Council of Medical Research (ICMR), New Delhi, for this doctoral study. The authors gratefully acknowledge the financial support from the Indian Council of Medical Research, New Delhi, India.

Role of funding source: This study was part of the doctoral research carried out at the National Institute of Mental Health and Neurosciences (NIMHANS), Bengaluru, India, and was funded by the Indian Council of Medical Research (ICMR), New Delhi (Ref No.: 3/1/3JRF-2011/HRD-104). No financial interests, direct or indirect, exist for the individual contributors in connection with the content of this paper. The funding body (ICMR) had no role in the study design; in the collection, analysis and interpretation of data; in the writing of the report; and in the decision to submit the article for publication.

Study registration: The study was registered with Clinical Trial Registry - India (Ref: CTRI/2017/08/ 009346). 
Design/methodology/approach - The sample comprised treatment-seeking alcohol-dependent persons $(n=50)$, allotted into two groups: (1) the treatment as usual (TAU) group $(n=25)$ and (2) the treatment group $(n=25)$ ]. The groups were matched on age $( \pm 1$ year $)$ and education $( \pm 1$ year). The TAU group received standard pharmacological treatment, psychotherapeutic sessions on relapse prevention and yoga for 18 days, while the treatment group received IIPA sessions in addition to the usual treatment. Auditory verbal learning test, complex figure test and $\mathrm{QoL}$ scale were administered at pre- and post-treatment along with screening measures.

Findings - The two groups were comparable on demographic variables, clinical characteristics and outcome measures at baseline. Pre- to post-treatment changes (gain scores) comparison between the treatment and TAU groups revealed a significant difference in verbal encoding, verbal and visual memory, verbal recognition, visuospatial construction and QoL.

Research limitations/implications - This study suggests that IIPA is effective for improving learning and memory in both modality (verbal and visual) and QoL in persons with alcoholism. The IIPA may help in better treatment recovery.

Practical implications - The IIPA may help in treatment for alcoholism and may enhance treatment efficacy.

Originality/value - IIPA is effective for improving learning and memory in both modalities and QoL in persons with alcohol dependence. The IIPA may help in better treatment recovery.

Keywords Alcohol dependence, Learning and memory, Quality of life, India

Paper type Research paper

\section{Introduction}

Alcohol-use disorder produces structural and functional brain dysfunctions, particularly in the frontal and limbic cortex $[1,2]$. Persons with alcohol dependence demonstrate multiple cognitive impairments such as executive function deficits (i.e. working memory, inhibitory control and cognitive flexibility), visuospatial abilities and learning and memory including episodic and prospective memory [3-5]. Some of the cognitive impairment caused by alcoholuse disorder is reversible with abstinence. However, several cognitive deficits, particularly visuospatial abilities, and memory functioning persist for several months to even more than one year of abstinence [6].

Cognitive deficits such as learning and memory in persons with alcohol dependence may contribute to everyday problems encountered even after treatment. Treatments for these individuals include motivational interviewing, relapse prevention and coping skills training. These skills help to manage the craving and prevent relapse and promote adaptive psychosocial functioning. In the treatment sessions, individuals are required to reflect on the negative consequences of alcohol dependence as well as the risky situations that could potentially induce craving and lead to drinking behavior. They are also expected to learn and remember the adaptive strategies to deal with craving effectively and to maintain sobriety [7]. To be able to effectively deal with these situations and apply the acquired skills and strategies, adequate learning and memory are essential. Learning and memory impairments are likely to affect adaptive behavioral change. Memory can predict readiness to change drinking behaviors [8].

Hence, individuals with cognitive impairments are less likely to benefit from the treatment of alcohol dependence. Cognitive impairments may lead to denial of the problems related to alcohol dependence, as individuals may fail to generalize from the examples, or relate the learning with experiences in treatment sessions. Thus, individuals may fail to benefit from experiences in treatment sessions. It may affect their motivation to actively participate in the treatment due to difficulties in comprehension [9].

Cognitive deficits may limit the person's ability to fully benefit from the treatment. Hence, it is imperative to address cognitive deficits, including memory in the treatment programs for alcohol dependence. Alcohol dependence adversely impacts a person's quality of life (QoL). QoL is a sense of well-being and satisfaction related to an individual's ability to function in daily life. Studies have reported considerably decreased QoL, which is an important aspect of
Integrated intervention program for alcoholism 
JHR

36,1

treatment $[10,11]$. It becomes imperative to consider QoL as an outcome measure because the individual's ability to function in daily life and overall sense of well-being and satisfaction is an important aspect of treatment - recovery [10].

Cognitive remediation is known to improve cognitive deficit recovery and psychosocial adjustment in persons with alcohol dependence [12]. Cognitive remediation has been proved effective in the treatment of substance-use disorders, including alcohol dependence $[13,14]$. We developed an integrated intervention program for alcoholism (IIPA) that consists of a cognitive remediation program and mind-body exercises [15]. The rationale for integrating cognitive remediation with mind-body exercises was based on neurocognitive theories of addiction $[16,17]$. Neurocognitive theories of addiction have emphasized that there is a need for intervention that enhances both executive functions and emotion regulation. The IIPA intends to address executive functions as well as emotion regulation, therefore attempts to promote the optimal balance between the prefrontal cortex-subcortical regions. This could facilitate the self-regulatory mechanisms and may prevent aberrant top-down (frontal) control of overwhelming bottom-up impulses from the limbic systems [15].

The cognitive remediation program comprises tasks to improve attention, working memory, mental flexibility and learning and memory. The underlying principle of cognitive remediation is neural plasticity [18]. Similarly, Qigong and Tai Chi Chuan are meditative exercises, originated from China [19]. They combine meditation with graceful movement, breathing and relaxation [20]. Both are known to enhance relaxation and reduce sympathetic nervous system activity [21,22] and improve cognitive functions, including memory [23, 24]. Multimodal intervention that includes cognitive remediation and mind-body exercise improves the resting-state connectivity between the medial prefrontal cortex $(\mathrm{mPFC})$ and medial temporal lobe (MTL) [25]. Mind-body exercise facilitates the resting-state functional connectivity between the hippocampus and $\mathrm{mPFC}$, increases gray matter volume at the left MTL and enhances encoding and retrieval of memory [26, 27]. Mind-body exercise also may facilitate neural/brain plasticity in regions implicated in drug addiction [28].

This study aimed to examine the effects of IIPA, which integrates cognitive remediation with mind-body exercise in improving verbal learning and memory, visuospatial construction, visual memory and QoL in persons with alcohol dependence.

\section{Methodology}

This study used a randomized matched case-control design with pre- and post-treatment assessment. Individuals diagnosed with alcohol dependence as per the diagnostic criteria of ICD-10 were recruited. All the participants had the early onset of alcohol dependence (alcohol dependence before 25 years of age) and positive family history of alcohol dependence (one or more first-degree family members with alcohol dependence). The exclusion criteria for the participants were: the positive history of other substance-use disorders (barring tobacco) such as cannabis, the positive history of major psychiatry disorders (i.e. schizophrenia and mood disorders), severe memory impairment as assessed on the Hindi Mental State Examination (score less than 24).

Participants were excluded from the study if they had received structured psychotherapy, meditation or cognitive retraining sessions in the previous year.

The sample was recruited from the inpatient services of the Centre for Addiction Medicine (CAM), National Institute of Mental Health and Neurosciences (NIMHANS), Bengaluru, India.

A total of 738 patients admitted to the CAM ward were approached and screened. Most of the patients were excluded due to late-onset alcohol dependence; poly-substance abuse; other substance abuse such as opioids, cannabis and benzodiazepine; comorbid of major psychiatric disorders such as schizophrenia; and affective disorders and/or complicated 
withdrawal. A detailed description can be seen in Kumar et al. [15]. Fifty participants completed both pre- and post-treatment assessments.

\section{Ethical consideration}

The study was approved by the Institutional Research and Ethics Committee (Ref:NIMH/DO/ SUB-COMMITTEE/2013) and was registered with Clinical Trial Registry India (Ref: CTRI/ 2017/08/009346).

\section{Measures}

Participant's demographic status and alcohol dependence-related information (such as the age of initiation, dependence of alcohol use and duration of alcohol use) were recorded on a socio-demographic datasheet. The Mini-international Neuropsychiatry Interview (MINI) v. 6.0 [29] was used as a screening tool for Axis I psychiatry disorders. Alcohol dependence in first-degree family members was documented on Family Interview for Genetic Studies (FIGS) [30]. Severe memory impairment was screened using Hindi Mental State Examination [31]. The Short Alcohol Dependence Data Questionnaire (SADD) [32] was administered for assessing the severity of alcohol dependence and Semi-Structured Assessment for Genetics of Alcoholism-version II (SSAGA-II) [33] was used to document externalizing spectrum disorders score.

\section{Auditory verbal learning test (AVLT) [34]}

This test was originally developed by Rey in 1958 as cited in [35]. The version used here is the World Health Organization and University of California at Los Angeles (WHO/UCLA) version, which was developed to reduce cultural bias [34]. This test has been standardized in the Indian context [36] and is widely used in clinical practice. A 15-item list of words (List A) was presented to the participant verbally, which were to be learned over five trials. Thereafter, a new 15-word list (List B) was presented to the participant as an interference word list. After the recall of the interference word list, the participant was asked to recall the 15 words (List A). This is taken as an immediate recall. Delayed recall of this List A was obtained after 20 min without prior warning to the participant, following which recognition phase was taken where the participant was verbally presented with the same 15 words (List A) along with new words. The task required participants to correctly identify words from List A and new words.

\section{Complex figure test (CFT)}

This test was first designed by Rey in 1941 as cited in [37]. The complex figure was placed in front of the participant along with the similar size of the one blank piece of paper. The participant was instructed to copy the figure without revealing the fact that he/she is supposed to recall the figure after a few minutes. The participant was asked to do the freehand drawing, without using a ruler to draw. An eraser was provided for erasing and correction purposes. The participant was asked to reproduce the design from memory after $3 \mathrm{~min}$ (immediate recall) and $30 \mathrm{~min}$ (delayed recall) without a prior warning. The intervening period was filled with other tasks. This test has been standardized in the Indian context [36] and it is widely used in clinical practice as well as in research study.

\section{Quality of life enjoyment and satisfaction questionnaire - short form (Q-LES-Q-SF) [38]}

The Quality of life enjoyment and satisfaction questionnaire - short form (Q-LES-Q-SF) is one of the most frequently used outcome measures in psychiatry research. The internal 
JHR

36,1

\section{4}

consistency and test-retest coefficients were reported as 0.9 and 0.93 , respectively. The Q-LES-Q-SF is reported to be $80 \%$ sensitive and $100 \%$ specific measure. The Q-LES-Q-SF is a reliable and valid clinical assessment tool for QoL [39]. It has items related to various aspects of QoL. Items are framed with a five-point rating scale, ranging from "Very Poor-1" to "Very Good-5." The total raw score can range from 14 to 70 . Subjects were asked to rate their QoL on five-point rating items during the previous week.

\section{Procedure}

All participants were inpatients at the CAM. They were detoxified with diazepam. Pretreatment assessment was carried out after 3-4 days of alcohol detoxification. Following baseline assessment, the treatment as usual (TAU) group received the usual treatment that included pharmacological treatment, sessions on relapse prevention (three sessions per week) and yoga (six sessions per week) for 18 days. The treatment group received an IIPA along with the usual treatment (except yoga) for 18 consecutive days. The IIPA has two treatment components: (1) cognitive remediation tasks and (2) mind-body exercises (Qigong and Tai Chi Chuan). Cognitive remediation tasks intend to ameliorate cognitive deficits or enhance cognitive functioning such as attention, working memory, mental flexibility, verbal learning and memory. Mind-body exercises (Qigong and Tai Chi Chuan) aimed to facilitate affect regulation, and alleviate stress, produce health benefits and improve overall well-being. More details about IIPA can be seen in a previously published manuscript [15]. Outcome measures of learning and memory (verbal and visual), as well as QoL, were administered again at posttreatment/usual treatment.

\section{Statistical analysis}

Data were analyzed using the Statistical Package for the Social Sciences v. 22 for Windows. Shapiro-Wilk's test showed that the data were normally distributed. Descriptive statistics were used. Differences between two groups such as socio-demographic characteristics and the clinical variables were analyzed using an independent sample $t$-test for continuous variables. Pre- to post-treatment changes (gain scores) were calculated for both groups and then compared between the groups with an independent sample $t$-test to see whether there were any significant differences. The effect size was described by Cohen's d.

\section{Results}

Participants were matched on age and education ( \pm 1 year). The average age was 34.28 years (range: 18-45 years; SD: 5.33) in the IIPA group and 34.08 years (range: $18-45$ years; SD: 5.73) in the TAU group. The average education was 11.08 years \pm 2.48 (SD) in the IIPA group and 11.12 years \pm 2.45 (SD) in the TAU group. The majority of participants from both the groups were from the middle socioeconomic status (56\% IIPA group, $44 \%$ TAU group) and the lower socioeconomic status (36\% IIPA group, $44 \%$ TAU group).

The two groups were compared on clinical variables related to alcoholism such as initiation age of alcohol use, age at which they became dependent and the duration of alcohol consumption. The results showed that there was no significant difference between the groups on initiation age of alcohol use [ $p=0.456$, IIPA: $18.64 \pm 2.53$; TAU: $19.24 \pm 3.09$ (mean \pm SD)], age at which they became dependent on alcohol $[p=0.662$, IIPA: $23.64 \pm 2.61$; TAU: $23.96 \pm 2.52]$, total years of alcohol use [ $p=0.812$, IIPA: $15.28 \pm 6.01$; TAU: $14.88 \pm 5.81]$, total years of alcohol dependence [ $p=0.680$, IIPA: $10.60 \pm 5.77$; TAU: $9.96 \pm 5.10]$, severity of alcohol use $[p=0.976$, IIPA: $23.24 \pm 8.66$; TAU: $23.32 \pm 10.29]$ and externalizing trait score $[p=0.069$, IIPA: $13.40 \pm 3.80$; TAU: $11.12 \pm 4.80]$. The two groups were comparable on pharmacotherapy [15]. 
Verbal learning and memory

An independent sample $t$-test showed that both groups were comparable on the verbal words encoding (learning trials) and verbal recognition (hit and false alarm) at baseline (Table 1). The TAU group had better verbal memory compared to the treatment (IIPA) group at baseline. Table 2, pre- to post-treatment scores (gain scores) showed that there was a significant difference between the IIPA and TAU groups on verbal encoding, verbal memory and verbal recognition. The mean of both groups indicates that the IIPA group had a significantly better improvement compared to the TAU group. The effect size was found to be large for verbal learning and memory.

\section{Visuospatial constructive ability and visual memory}

There was no significant difference between the two groups at baseline (Table 1). Pre-to posttreatment scores were calculated for both groups, and the results showed that there was a significant difference between the IIPA and TAU groups on visuospatial construction and visual memory (Table 2). The IIPA group showed significantly better improvement compared to the TAU group. The effect size was found to be large for visual memory.

\section{Quality of life}

Table 1, there was no significant difference between the groups on the total score of QoL at baseline. Pre-to post-treatment score was calculated for both groups. There was a significant difference between the IIPA and TAU groups on QoL where the IIPA group had significantly better improvement compared to the TAU group, Table 2. The effect size was found to be large.

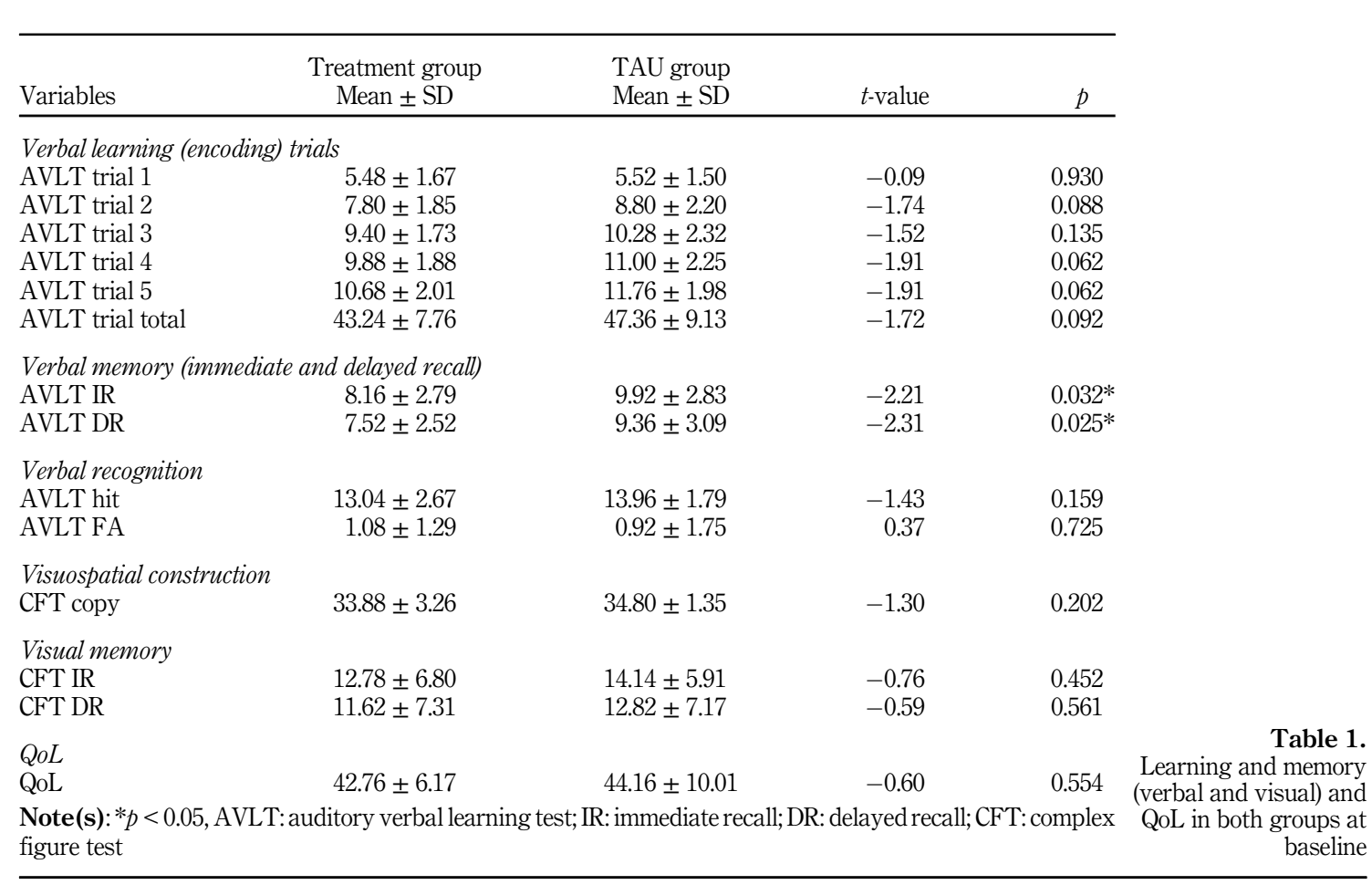

Note(s): $* p<0.05$, AVLT: auditory verbal learning test; IR: immediate recall; DR: delayed recall; CFT: complex figure test
Integrated intervention program for alcoholism 


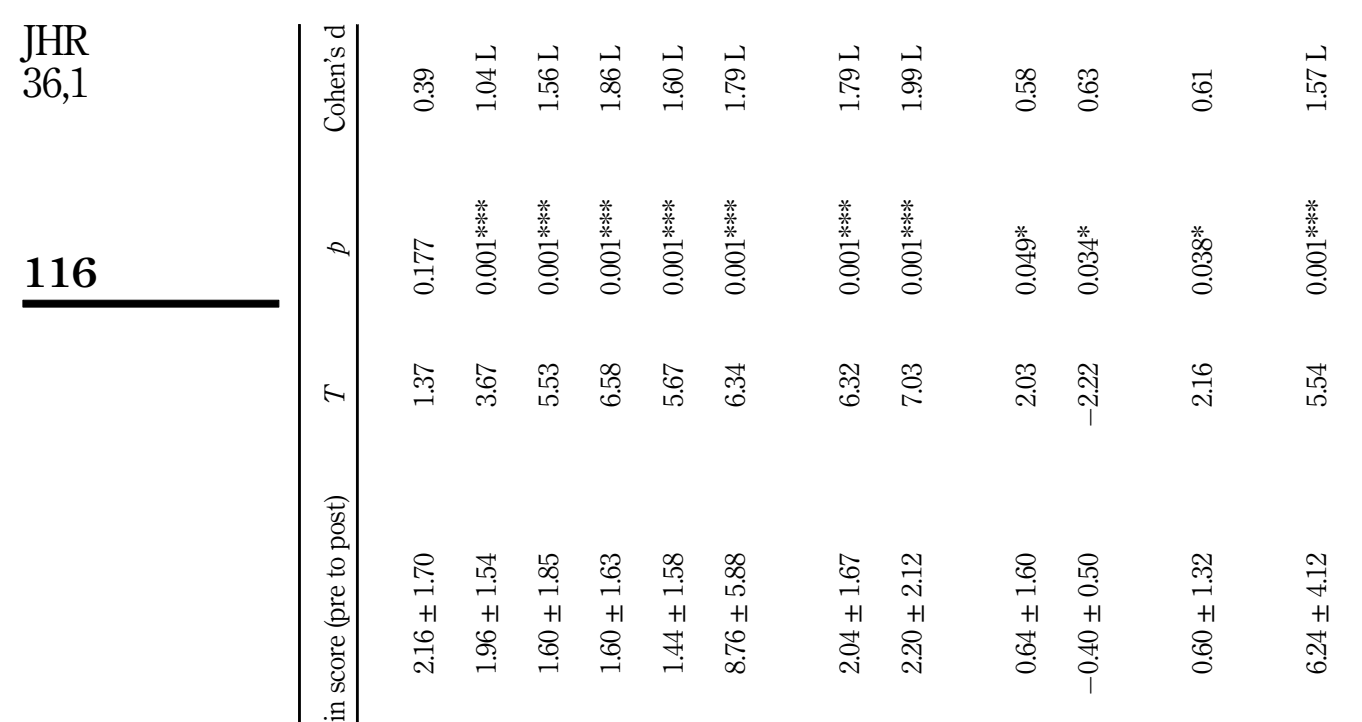

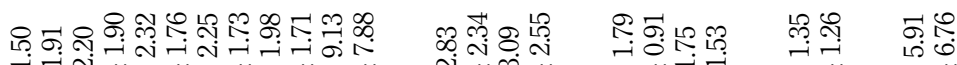

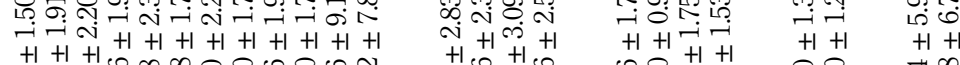

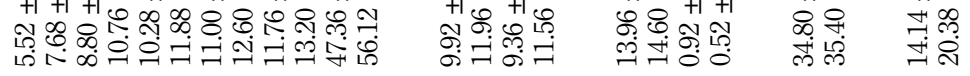
|| || || || || || || || || || || || || || || || || || || || || || || ||

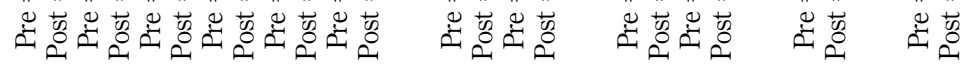

Table 2.

Gain score from pre- to post-intervention in both groups and between-group change score

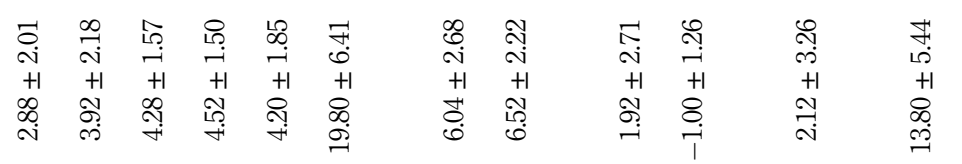

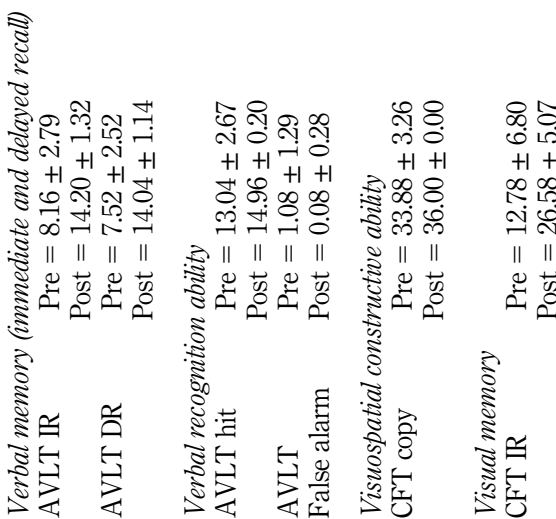


JHR

36,1

118

\section{Discussion}

Psychosocial treatments for persons with alcohol dependence such as motivational interviewing and relapse prevention focus on changing drinking behaviors, developing skills to prevent relapse and promote adaptive psychosocial functioning [7]. In the treatment, individuals are required to reflect on previous and probable future negative consequences of alcoholism, remembering the risky situations and contexts that can induce craving and lead to drinking behavior. They were expected to learn and remember the adaptive strategies to deal with craving effectively and to maintain sobriety. Learning and memory impairments can adversely impact the patient's grasping and remembering capacity. To be able to effectively deal with these situations and apply the acquired skills and strategies, adequate learning and memory are essential. Hence, learning and memory impairments are likely to affect the adaptive behavioral change in persons with alcohol dependence. Learning and memory deficits may not be ameliorated with the usual treatment of alcoholism [6]. It becomes imperative that the treatment programs for alcoholism need to address these cognitive deficits. So, specific interventions are required to ameliorate neurocognitive deficits, and the IIPA is one such intervention developed specifically for treating alcohol-related neurocognitive deficits.

The findings indicated that there was a significant difference between the treatment (IIPA) and the TAU group on learning and memory in both modalities. The treatment (IIPA) group had comparatively better improvement from pre- to post-treatment. The effect size was also found to be large. The IIPA has two components, cognitive remediation tasks (such as attention, executive functions, learning and memory) that intend to improve cognitive functioning and mind-body exercises that intend to improve relaxation, emotion regulation and cognitive functioning [15].

Substance-use disorders, including alcohol dependence, produce impairment in cognitive control by affecting the central nervous system, and poor self-control can lead the alcoholdependent person to succumb to craving and relapse [40]. When an individual tries to abstain from alcohol dependence, the physical and mental symptoms that result from the withdrawal syndrome increase the risk of relapse [41]. The mind-body exercises are beneficial in reducing various drug withdrawal symptoms [42]. Tai Chi and Qigong could improve the affective state in persons with substance-use disorders and enhance various dimensions of health and QoL, as they have meditative components [43]. Tai Chi and Qigong exercise can reduce physiological arousal and increase relaxation, which may help in relapse prevention [44]. The Tai Chi and Qigong exercises can facilitate prefrontal regulation which ultimately facilitates the cognitive control that is essential in substance-use disorders [44]. Mind-body exercises because of their meditative aspect can generate new synaptic connections in the prefrontal lobe and strengthen its functioning. While, it decreases limbic activation and thus contributes to emotion regulation [45].

Furthermore, Tai Chi and Qigong exercises may facilitate physical as well as cognitive stimulation, as these exercises require coordinated bodily movements, balance and conscious attention to each movement [46]. Tai Chi practice induces mental alertness that helps in preventing omissions and avoiding mistakes/errors [47]. Tai Chi and Qigong can improve attention in dual ways by the eye focusing on bodily movement and the mind focusing on breathing and movement sequences [48]. The mechanism of change in cognitive functions from exercise-based intervention is reported to be due to changes in white matter and gray matter volume [49], as well as increased functional connectivity and improvement in executive functions $[49,50]$. Exercise facilitates synaptic plasticity and neurogenesis [51, 52]. Exercisebased intervention can increase hippocampal volume and thus improve memory [53].

Similarly, studies $[13,14]$ have demonstrated the effectiveness of cognitive remediation in improving cognitive functioning in persons with alcohol dependence. Executive functions are known to facilitate encoding (learning), in terms of strategic organization and retrieval [54, 55]. 
The cognitive remediation program included in the IIPA has several tasks (such as attention enhancement task, encoding and error detection task, working memory task and learning and memory task) to facilitate cognitive functioning including learning and memory.

The results indicated that IIPA led to relatively better improvement in QoL. Improvement in QoL is an important characteristic of recovery processes in persons with substance-use disorders, including alcohol dependence $[10,11]$. This can be used as a motivation tool to mark recovery and treatment efficacy [56]. The augmented improvement in the QoL in the treatment group could be due to improved executive functions, which might have led to better self-regulation in behavior and thought as well as better daily functioning. On the other hand, the practice of Tai Chi and Qigong could have also produced a stress-relieving experience, better sleep, positive mood state and health benefits $[57,58]$. Thus, the reduction of the negative emotional state and relieving effect of stress may have facilitated a better QoL.

\section{Limitations of this study}

This were a small sample size and lack of follow-up assessment of outcome variables, which could have provided more information about the maintenance of improvement. There is a possibility that other types of memory such as episodic memory, which was not included in the current study, might be impaired in persons with alcohol dependence [59]. Future studies may examine IIPA effects on various other types of memory implicated in persons with alcohol dependence.

Conflict of interest: There is no conflict of interest.

\section{References}

1. Moselhy HF, Georgiou G, Kahn A. Frontal lobe changes in alcoholism: a review of the literature. Alcohol Alcohol. 2001; 36(5): 357-68. doi: 10.1093/alcalc/36.5.357.

2. Vabret F, Lannuzel C, Cabe N, Ritz L, Boudehent C, Eustache F, et al. Alcohol-related neuropsychological impairments: nature, impact and detection. Presse Med. 2016; $45(12 \mathrm{Pt}$ 1): 1124-32. doi: 10.1016/j.lpm.2016.01.030.

3. Le Berre AP, Fama R, Sullivan EV. Executive functions, memory, and social cognitive deficits and recovery in chronic alcoholism: a critical review to inform future research. Alcohol Clin Exp Res. 2017; 41(8): 1432-43. doi: 10.1111/acer.13431.

4. Kopera M, Wojnar M, Brower K, Glass J, Nowosad I, Gmaj B, et al. Cognitive functions in abstinent alcohol-dependent patients. Alcohol. 2012; 46(7): 665-71. doi: 10.1016/j.alcohol.2012.04.005.

5. van Holst RJ, Schilt T. Drug-related decrease in neuropsychological functions of abstinent drug users. Curr Drug Abuse Rev. 2011; 4(1): 42-56. doi: 10.2174/1874473711104010042.

6. Crowe SF, Cammisuli DM, Stranks EK. Widespread cognitive deficits in alcoholism persistent following prolonged abstinence: an updated meta-analysis of studies that used standardised neuropsychological assessment tools. Arch Clin Neuropsychol. 2019; 35(1): 31-45. doi: 10.1093/ arclin/acy106.

7. Pitel AL, Beaunieux H, Witkowski T, Vabret F, Guillery-Girard B, Quinette P, et al. Genuine episodic memory deficits and executive dysfunctions in alcoholic subjects early in abstinence. Alcohol Clin Exp Res. 2007; 31(7): 1169-78. doi: 10.1111/j.1530-0277.2007.00418.x.

8. Blume AW, Schmaling KB, Marlatt GA. Memory, executive cognitive function, and readiness to change drinking behavior. Addict Behav. 2005; 30(2): 301-14. doi: 10.1016/j.addbeh.2004.05.019.

9. Fals-Stewart W, Shanahan T, Brown L. Treating alcoholism and substance abuse: a neuropsychiatric perspective. Psychother Priv Pact. 1995; 14(1): 1-21. doi: 10.1300/J294v14n01_01.

10. Donovan D, Mattson ME, Cisler RA, Longabaugh R, Zweben A. Quality of life as an outcome measure in alcoholism treatment research. J Stud Alcohol Suppl. 2005; 15: 119-39. discussion 92-3. doi: 10.15288/jsas.2005.s15.119. 
JHR

36,1

11. Foster JH, Peters TJ, Marshall EJ. Quality of life measures and outcome in alcohol-dependent men and women. Alcohol. 2000; 22(1): 45-52. doi: 10.1016/s0741-8329(00)00102-6.

12. Cunha PJ, Novaes MA. Neurocognitive assessment in alcohol abuse and dependence: implications for treatment. Braz J Psychiatry. 2004; 26(Suppl 1): S23-7. doi: 10.1590/s151644462004000500007.

13. Rupp CI, Kemmler G, Kurz M, Hinterhuber H, Fleischhacker WW. Cognitive remediation therapy during treatment for alcohol dependence. J Stud Alcohol Drugs. 2012; 73(4): 625-34. doi: 10.15288/ jsad.2012.73.625.

14. Gamito P, Oliveira J, Lopes P, Brito R, Morais D, Silva D, et al. Executive functioning in alcoholics following an mHealth cognitive stimulation program: randomized controlled trial. J Med Internet Res. 2014; 16(4): e102. doi: 10.2196/jmir.2923.

15. Kumar R, Kumar KJ, Benegal V, Roopesh BN, Ravi GS. Effectiveness of an integrated intervention program for alcoholism (IIPA) for enhancing self-regulation: preliminary evidence. Asian J Psychiatr. 2019; 43: 37-44. doi: 10.1016/j.ajp.2019.05.006.

16. Heatherton TF. Neuroscience of self and self-regulation. Annu Rev Psychol. 2011; 62: 363-90. doi: 10.1146/annurev.psych.121208.131616.

17. Verdejo-Garcia A, Bechara A. A somatic marker theory of addiction. Neuropharmacology. 2009; 56(Suppl 1): 48-62. doi: 10.1016/j.neuropharm.2008.07.035.

18. Keshavan MS, Vinogradov S, Rumsey J, Sherrill J, Wagner A. Cognitive training in mental disorders: update and future directions. Am J Psychiatry. 2014; 171(5): 510-22. doi: 10.1176/appi. ajp.2013.13081075.

19. Birdee GS, Wayne PM, Davis RB, Phillips RS, Yeh GY. T'ai Chi and Qigong for health: patterns of use in the United States. J Altern Complement Med. 2009; 15(9): 969-73. doi: 10.1089/acm. 2009.0174.

20. Wang C, Schmid CH, Rones R, Kalish R, Yinh J, Goldenberg DL, et al. A randomized trial of tai chi for fibromyalgia. N Engl J Med. 2010; 363(8): 743-54. doi: 10.1056/NEJMoa0912611.

21. Motivala SJ, Sollers J, Thayer J, Irwin MR. Tai Chi Chih acutely decreases sympathetic nervous system activity in older adults. J Gerontol A Biol Sci Med Sci. 2006; 61(11): 1177-80. doi: 10.1093/ gerona/61.11.1177.

22. Irwin MR, Olmstead R, Motivala SJ. Improving sleep quality in older adults with moderate sleep complaints: a randomized controlled trial of Tai Chi Chih. Sleep. 2008; 31(7): 1001-8.

23. Lam LC, Chau RC, Wong BM, Fung AW, Lui VW, Tam CC, et al. Interim follow-up of a randomized controlled trial comparing Chinese style mind body (Tai Chi) and stretching exercises on cognitive function in subjects at risk of progressive cognitive decline. Int J Geriatr Psychiatry. 2011; 26(7): 733-40. doi: 10.1002/gps.2602.

24. Tao J, Chen X, Liu J, Egorova N, Xue X, Liu W, et al. Tai Chi Chuan and Baduanjin mindbody training changes resting-state low-frequency fluctuations in the frontal lobe of older adults: a resting-state fMRI study. Front Hum Neurosci. 2017; 11: 514. doi: 10.3389/fnhum.2017.00514.

25. Li R, Zhu X, Yin S, Niu Y, Zheng Z, Huang X, et al. Multimodal intervention in older adults improves resting-state functional connectivity between the medial prefrontal cortex and medial temporal lobe. Front Aging Neurosci. 2014; 6: 39. doi: 10.3389/fnagi.2014.00039.

26. Tao J, Liu J, Liu W, Huang J, Xue X, Chen X, et al. Tai Chi Chuan and Baduanjin increase grey matter volume in older adults: a brain imaging study. J Alzheimers Dis. 2017; 60(2): 389-400. doi: 10.3233/JAD-170477.

27. Tao J, Liu J, Egorova N, Chen X, Sun S, Xue X, et al. Increased hippocampus-medial prefrontal cortex resting-state functional connectivity and memory function after Tai Chi Chuan practice in elder adults. Front Aging Neurosci. 2016; 8: 25. doi: 10.3389/fnagi.2016.00025.

28. Gomez-Pinilla F, Zhuang Y, Feng J, Ying Z, Fan G. Exercise impacts brain-derived neurotrophic factor plasticity by engaging mechanisms of epigenetic regulation. Eur J Neurosci. 2011; 33(3): 383-90. doi: 10.1111/j.1460-9568.2010.07508.x. 
29. Sheehan DV, Lecrubier Y, Sheehan KH, Amorim P, Janavs J, Weiller E, et al. The MiniInternational Neuropsychiatric Interview (M.I.N.I.): the development and validation of a structured diagnostic psychiatric interview for DSM-IV and ICD-10. J Clin Psychiatry. 1998; 59(Suppl 20): 22-33 [quiz 4-57].

30. Maxwell ME. Family interview for genetic studies (FIGS): a manual for FIGS. In: Neurogenetics branch, intramural research program. Bethesda, MD: ClinicalNational Institute of Mental Health; 1992.

31. Ganguli M, Ratcliff G, Chandra V, Sharma S, Gilby J, Pandav R, et al. A Hindi version of the MMUE - the development of a cognitive screening instrument for a largely illiterate rural elderly population in India. Int J Geriatr Psychiatry. 1995; 10(5): 367-77. doi: 10.1002/gps.930100505.

32. Raistrick D, Dunbar G, Davidson R. Development of a questionnaire to measure alcohol dependence. Br J Addict. 1983; 78(1): 89-95.

33. Bucholz KK, Cadoret R, Cloninger CR, Dinwiddie SH, Hesselbrock VM, Nurnberger JI Jr, et al. A new, semi-structured psychiatric interview for use in genetic linkage studies: a report on the reliability of the SSAGA. J Stud Alcohol. 1994; 55(2): 149-58. doi: 10.15288/jsa. 1994.55.149.

34. Maj M, D'Elia L, Satz P, Janssen R, Zaudig M, Uchiyama C, et al. Evaluation of two new neuropsychological tests designed to minimize cultural bias in the assessment of HIV-1 seropositive persons: a WHO study. Arch Clin Neuropsychol. 1993; 8(2): 123-35.

35. Spreen O, Strauss E. A compendium of neuropsychological tests: administration, norms, and commentary. 2nd ed. New York: Oxford University Press; 1998.

36. Rao SL, Subbakrishna DK, Gopukumar K. NIMHANS neuropsychology battery-2004, manual. Bangalore: National Institute of Mental Health and Neurosciences; 2004.

37. Meyers JE, Meyer KR. Rey complex figure test and recognition trial: professional manual. Odessa: Psychological Assessment Resources; 1995.

38. Endicott J, Nee J, Harrison W, Blumenthal R. Quality of life enjoyment and satisfaction questionnaire: a new measure. Psychopharmacol Bull. 1993; 29(2): 321-6.

39. Stevanovic D. Quality of life enjoyment and satisfaction questionnaire-short form for quality of life assessments in clinical practice: a psychometric study. J Psychiatr Ment Health Nurs. 2011; 18(8): 744-50. doi: 10.1111/j.1365-2850.2011.01735.x.

40. Belin D, Belin-Rauscent A, Murray JE, Everitt BJ. Addiction: failure of control over maladaptive incentive habits. Curr Opin Neurobiol. 2013; 23(4): 564-72. doi: 10.1016/j.conb.2013.01.025.

41. Baker TB, Piper ME, McCarthy DE, Majeskie MR, Fiore MC. Addiction motivation reformulated: an affective processing model of negative reinforcement. Psychol Rev. 2004; 111(1): 33-51. doi: 10. 1037/0033-295X.111.1.33.

42. Brown RA, Abrantes AM, Read JP, Marcus BH, Jakicic J, Strong DR, et al. Aerobic exercise for alcohol recovery: rationale, program description, and preliminary findings. Behav Modif. 2009; 33(2): 220-49. doi: 10.1177/0145445508329112.

43. Polonen P, Lappi O, Tervaniemi M. Effect of meditative movement on affect and flow in Qigong practitioners. Front Psychol. 2019; 10: 2375. doi: 10.3389/fpsyg.2019.02375.

44. Priddy SE, Howard MO, Hanley AW, Riquino MR, Friberg-Felsted K, Garland EL. Mindfulness meditation in the treatment of substance use disorders and preventing future relapse: neurocognitive mechanisms and clinical implications. Subst Abuse Rehabil. 2018; 9: 103-14. doi: 10.2147/SAR.S145201.

45. Tang YY. Mechanism of integrative body-mind training. Neurosci Bull. 2011; 27(6): 383-8. doi: 10. 1007/s12264-011-1141-2.

46. Lam LC, Chau RC, Wong BM, Fung AW, Tam CW, Leung GT, et al. A 1-year randomized controlled trial comparing mind body exercise (Tai Chi) with stretching and toning exercise on cognitive function in older Chinese adults at risk of cognitive decline. J Am Med Dir Assoc. 2012; 13(6): 568-20. doi: 10.1016/j.jamda.2012.03.008.
Integrated intervention program for alcoholism 
JHR

36,1

122
47. Kim TH, Pascual-Leone J, Johnson J, Tamim H. The mental-attention Tai Chi effect with older adults. BMC Psychol. 2016; 4(1): 29. doi: 10.1186/s40359-016-0137-0.

48. Wayne PM, Buring JE, Davis RB, Connors EM, Bonato P, Patritti B, et al. Tai Chi for osteopenic women: design and rationale of a pragmatic randomized controlled trial. BMC Musculoskelet Disord. 2010; 11: 40. doi: 10.1186/1471-2474-11-40.

49. Erickson KI, Leckie RL, Weinstein AM. Physical activity, fitness, and gray matter volume. Neurobiol. Aging. 2014; 35(Suppl 2): S20-8. doi: 10.1016/j.neurobiolaging.2014.03.034.

50. Voss MW, Prakash RS, Erickson KI, Basak C, Chaddock L, Kim JS, et al. Plasticity of brain networks in a randomized intervention trial of exercise training in older adults. Front Aging Neurosci. 2010; 2. doi: 10.3389/fnagi.2010.00032.

51. van Praag H. Neurogenesis and exercise: past and future directions. Neuromolecular Med. 2008; 10(2): 128-40. doi: 10.1007/s12017-008-8028-z.

52. Gomez-Pinilla F, Hillman C. The influence of exercise on cognitive abilities. Compr Physiol. 2013; 3(1): 403-28. doi: 10.1002/cphy.c110063.

53. Erickson KI, Voss MW, Prakash RS, Basak C, Szabo A, Chaddock L, et al. Exercise training increases size of hippocampus and improves memory. Proc Natl Acad Sci USA. 2011; 108(7): 3017-22. doi: 10.1073/pnas.1015950108.

54. Kapur S, Craik FI, Tulving E, Wilson AA, Houle S, Brown GM. Neuroanatomical correlates of encoding in episodic memory: levels of processing effect. Proc Natl Acad Sci USA. 1994; 91(6): 2008-11. doi: 10.1073/pnas.91.6.2008.

55. Habib R, McIntosh AR, Wheeler MA, Tulving E. Memory encoding and hippocampally-based novelty/familiarity discrimination networks. Neuropsychologia. 2003; 41(3): 271-9. doi: 10.1016/ s0028-3932(02)00160-4.

56. Ugochukwu C, Bagot KS, Delaloye S, Pi S, Vien L, Garvey T, et al. The importance of quality of life in patients with alcohol abuse and dependence. Harv Rev Psychiatry. 2013; 21(1): 1-17. doi: 10. 1097/HRP.0b013e31827fd8aa.

57. Li M, Fang Q, Li J, Zheng X, Tao J, Yan X, et al. The effect of Chinese traditional exerciseBaduanjin on physical and psychological well-being of college students: a randomized controlled trial. PLoS One. 2015; 10(7): e0130544. doi: 10.1371/journal.pone.0130544.

58. Chan JS, Ho RT, Chung KF, Wang CW, Yao TJ, Ng SM, et al. Qigong exercise alleviates fatigue, anxiety, and depressive symptoms, improves sleep quality, and shortens sleep latency in persons with chronic fatigue syndrome-like illness. Evid Based Complement Alternat Med. 2014; 2014: 106048. doi: $10.1155 / 2014 / 106048$.

59. Le Berre AP, Pinon K, Vabret F, Pitel AL, Allain P, Eustache F, et al. Study of metamemory in patients with chronic alcoholism using a feeling-of-knowing episodic memory task. Alcohol Clin Exp Res. 2010; 34(11): 1888-98. doi: 10.1111/j.1530-0277.2010.01277.x.

\section{Corresponding author}

Rajesh Kumar can be contacted at: bhurajes@gmail.com

For instructions on how to order reprints of this article, please visit our website:

www.emeraldgrouppublishing.com/licensing/reprints.htm

Or contact us for further details: permissions@emeraldinsight.com 